\title{
Quasi-phase-matched high harmonic generation in structured plasmas
}

\author{
Michael Wöstmann ${ }^{1, *}$, Lukas Splitthoff ${ }^{1}$, and Helmut Zacharias ${ }^{1}$ \\ ${ }^{1}$ Center for Nanotechnology, Heisenbergtraße 11, 48149 Münster, Germany
}

\begin{abstract}
Structured plasmas are employed in order to realize the quasiphase-matched generation of high harmonics. The plasma originates from laser ablated solids leading to the appearance of multiple separated jets. By altering the shape of the solid the dimensions of the plasma structure are easily optimized. Combining optimal jet widths and spacing the maximum enhancement of the harmonic intensity by a factor of 16 is observed after four plasma jets.
\end{abstract}

\section{Introduction}

High-harmonic generation (HHG) is an important process in attosecond-science [1]. However, only about a factor of $10^{-6}$ of the power of the initial driving radiation is converted into a single harmonic in conventional HHG setups [2]. Therefore contrast and signal-to-noise ratios are limited in applications. Phase-matched sources using waveguides have the prospect of an increased irradiated power [3]. However, they deal with low ionization fractions. Therefore the density of radiation emitting atoms is limited, especially for the highest harmonic orders. For that reason quasi-phase-matched sources have been implemented. They are enabled by either an intensity modulation of the driving beam in a waveguide [4] or a direct modulation of the density of the interaction medium [5]. The latter has been transferred to HHG in laser-ablated plasmas as well $[6,7]$. Since the plasma formation is dependent on the shape and spatial dimensions of a solid target, different plasma extensions and modulations are easily realizable.

Three contributions lead to a phase-mismatch in spatially extended interaction media. These are the geometrical phase advance of the driving beam through a focus (Gouy phase), the phase slip of the generated harmonic field caused by the intensity variation of the driving field, and dispersion of two electromagnetic fields inside a plasma. For the short trajectories the second contribution is negligible in the presented studies. Away from resonances, dispersion is generally dominated by free electrons in plasmas. They increase the phase-mismatch caused by the Gouy phase of the driving beam as their contributions are of the same sign. Therefore the phase-mismatch advances faster at high plasma densities inside the jets than in the gaps between them where the plasma density vanishes. This circumstance will result in shorter plasma jet lengths compared to their separation for optimal quasi-phase-matching.

\footnotetext{
*Corresponding author: michael.woestmann@wwu.de
} 


\section{Experimental realization}

A defined plasma structure is realized by illuminating a structured aluminum target with laser radiation. The plasma is excited on the surfaces of separated portions of stacked target material with their width and spacing selectable. Because the laser system operates at $1 \mathrm{kHz}$ the target needs to be rotating. Therefore round disks and spacers are applied with the axis of rotation parallel to the HHG driving beam direction. The latter is focused along the surfaces of the disks and passes through the individual plasma jets.

The laser source is a Ti:sapphire laser system operating at a central wavelength of $800 \mathrm{~nm}$. For the frequency conversion pulses of $40 \mathrm{fs}$ pulse duration are spherically focused with a Rayleigh length of $\mathrm{Z}_{\mathrm{R}}=0.5 \mathrm{~cm}$ and a beam waist radius of $\mathrm{w}_{0}=38 \mu \mathrm{m}$ approximately in the middle of the target. The peak intensity amounts to $1.1 \times 10^{14} \mathrm{~W} \mathrm{~cm}$. For the plasma formation uncompressed radiation of the same laser is cylindrically focused onto the face surface of the target with a peak intensity of $1.9 \times 10^{10} \mathrm{~W} \mathrm{~cm}{ }^{-2}$. A delay of $40 \mathrm{~ns}$ between the plasma producing and driving pulses ensures for proper plasma expansion away from the target surface. The generated harmonics are dispersed using a flat-field grating. Afterwards they are directed to a multichannel plate / phosphor screen combination and detected by a CCD.

\section{Results}

Without knowing the free electron density inside a plasma the optimal width of the individual short plasma jets for quasi-phase-matching a specific harmonic order can still be evaluated experimentally. This is realized by applying an unstructured target which is capable to create a broad unmodulated plasma. When the plasma width is varied, the coherence lengths can be determined directly. In these studies a knife edge in the plasma producing beam is used in order to reduce its width and thereby the extension of the plasma. As a result a sinusoidal dependence of the harmonic intensity on the plasma length is observed. The resulting coherence lengths are decreasing with increasing harmonic order as the phase-mismatch advances faster. For harmonics 17 to 23 the coherence lengths of the Al plasma are shown in Fig. 1 (a).
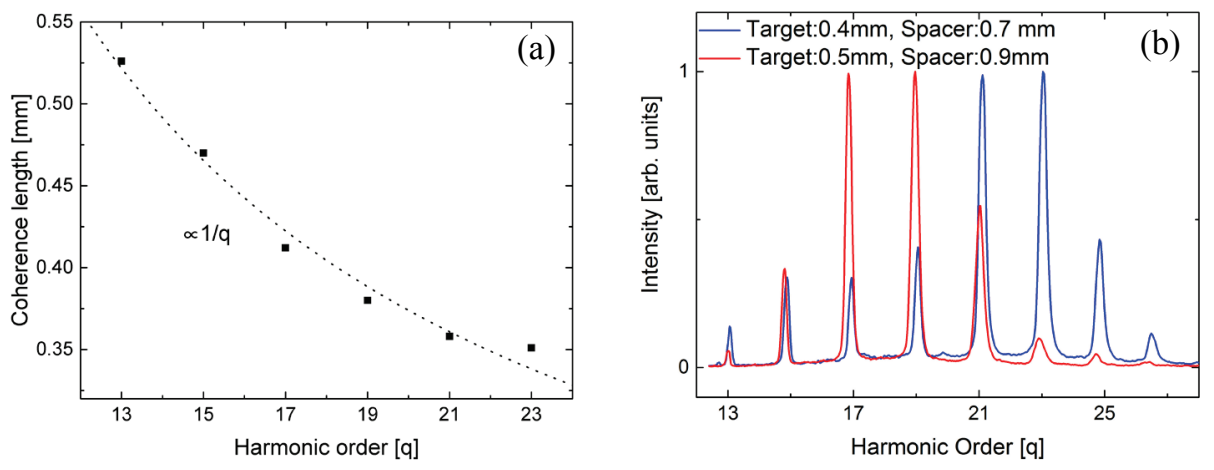

Fig. 1. Experimentally determined coherence lengths for different harmonic orders (a). Intensity of different harmonic orders employing different disk widths and spacing (b).

The optimal spacing of the target disks is determined from the Gouy phase of the driving beam. For the applied focusing geometry this is the only relevant contribution to a phase-mismatch outside the plasma jets. By properly structuring the target any desired range of harmonic orders can be enhanced. In Fig. 1 (b) two examples are presented with different target geometries. $0.4 \mathrm{~mm}$ wide targets favor the $21 \mathrm{st}$ and $23 \mathrm{rd}$ harmonic orders 
whereas $0.5 \mathrm{~mm}$ wide targets favor the 17 th and 19th orders. The optimal target disk widths are about $0.05-0.1 \mathrm{~mm}$ wider than the experimentally measured coherence lengths. This may result from plasma extension into the vacuum at the sides of the individual target disks.

Using four disks of $0.4 \mathrm{~mm}$ width and $0.7 \mathrm{~mm}$ pitch the harmonic intensity reveals a staircase-like profile as function of the plasma producing beam width when quasi-phasematching is optimized. This is shown in Fig 2 (a). The effective plasma width is plotted versus intensity in Fig. 2 (c). For the 23rd and 25th harmonic the curve follows a quadratic dependence characteristic for a phase-matched source. The intensity of the 23 rd harmonic is enhanced by the highest possible factor of 16 compared to the attainable intensity in unmodulated plasmas. Further details are to be found in [8].
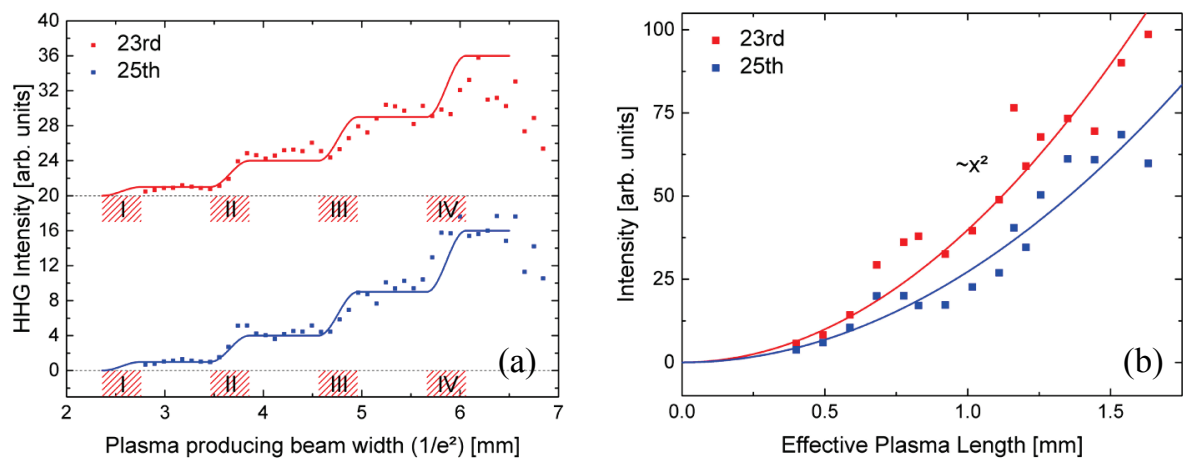

Fig. 2. Dependence of the intensity on the width of the plasma producing beam (a) and the effective plasma length showing the quadratic dependence of a phase-matched source (b). The target dimensions are $0.4 \mathrm{~mm}$ target disks and $0.7 \mathrm{~mm}$ spacing. The intensity is normalized to the maximal intensity of the 23rd harmonic generated in an unstructured plasma.

\section{Conclusion}

Quasi-phase-matched generation of high harmonics is realized and controlled by properly structuring a plasma that is excited from the surface of an aluminum target. By employing different target dimensions different ranges of harmonic orders are enhanced.

\section{References}

1. F. Krausz, M. Ivanov, Rev. Mod. Phys. 81, 163 (2009)

2. S. Hädrich, A. Klenke, J. Rothhardt, M. Krebs, A. Hoffmann, O. Pronin, V. Pervak, J. Limpert, A. Tünnermann, Nat. Photonics 8, 779 (2014)

3. A. Rundquist, C.G. Durfee III, Z. Chang, C. Herne, S. Backus, M.M. Murnane, H.C. Kapteyn, Science 280, 1412 (1998)

4. A. Paul, R.A. Bartels, R. Tobey, H. Green, S. Weiman, I.P. Christov, M M. Murnane, H.C. Kapteyn, S. Backus, Nature 421, 51 (2003)

5. J. Seres, V.S. Yakovlev, E. Seres, C. Streli, P. Wobrauschek, C. Spielmann, F. Krausz, Nat. Phys. 3, 878 (2007)

6. R.A. Ganeev, M. Suzuki, H. Kuroda, Phys. Rev. A 89, 033821 (2014)

7. V.V. Strelkov, R.A. Ganeev, Opt. Express 25, 21068 (2017)

8. M. Wöstmann, L. Splitthoff, H. Zacharias, Opt. Express 26, 14524 (2018) 